\title{
Subacute combined degeneration: clinical, electrophysiological, and magnetic resonance imaging findings
}

\author{
B Hemmer, F X Glocker, M Schumacher, G Deuschl, C H Lücking
}

\begin{abstract}
Objective-Vitamin $B_{12}$ deficiency is a systemic disease that often affects the nervous system. One of the most prevalent manifestations is subacute combined degeneration (SCD) of the spinal cord. To access the clinical, electrophysiological, and structural abnormalities associated with SCD, a study was conducted in nine patients.
\end{abstract}

Methods-Clinical, electrophysiological (electroneurography, somatosensory and motor evoked potentials), and MRI evaluations were performed in patients before and after treatment.

Results-The most prominent clinical and electrophysiological findings in all patients were dysfunctions of the posterior column. Corresponding hyperintense lesions in the posterior column of the spinal cord were found in two patients by $T 2$ weighted MRI. Damage to the central motor pathway was identified in four patients. Demyelinating neuropathy was present in one patient and axonal neuropathy in four. All patients showed improvement of their symptoms after treatment with cobalamin. Abnormalities of the spinal cord on MRI disappeared early in recovery. Motor evoked potentials and median somatosensory evoked potentials typically normalised after treatment, whereas tibial somatosensory evoked potentials remained abnormal in most patients.

Conclusions-Clinical, electrophysiological, and MRI findings associated with SCD in vitamin $B_{12}$ deficiency are diverse. Thus vitamin $B_{12}$ deficiency should be considered in the differential diagnosis of all spinal cord, peripheral nerve, and neuropsychiatric disorders.

(F Neurol Neurosurg Psychiatry 1998;65:822-827)

Keywords: vitamin $B_{12}$ deficiency; subacute combined degeneration; myelopathy

Vitamin $\mathrm{B}_{12}$ deficiency causes a wide range of haematological, gastrointestinal, psychiatric, and neurological disorders. ${ }^{1}$ Megaloblastic anaemia is a common early symptom leading to the diagnosis, although neurological symptoms may occur in the absence of haematological abnormalities. $^{2}$ The neurological syndromes associated with vitamin $B_{12}$ deficiency include myelopathy, neuropathy, neuropsychiatric abnormalities, and less often, optic nerve atrophy.
The spinal cord affliction called subacute combined degeneration (SCD) is clinically characterised by symmetric dysaesthesia, disturbance of the position sense, and spastic paraparesis or tetraparesis. ${ }^{1}$ Although most patients respond well to cobalamin treatment, residual neurological abnormalities persist in most. ${ }^{3}$ Studies involving limited numbers of patients with cobalamin deficiency have shown various abnormal electrophysiological findings ${ }^{4-9}$ and more recently lesions of the cervical spinal cord by MRI. ${ }^{10-12}$

Here, we present clinical, electrophysiological, and MRI findings of nine patients affected by SCD. Seven of these patients were followed up after treatment.

Materials and methods

The retrospective study was carried out between 1989 and 1996. Our report includes patients meeting all of the following criteria: low vitamin $\mathrm{B}_{12}$ serum concentrations or abnormal Schilling test, clinical signs of myelopathy, clinical improvement after cobalamin treatment, and no other spinal cord or peripheral nerve disease.

Routine blood chemistry, blood cell, and vitamin $\mathrm{B}_{12}$ serum concentration analyses were performed in all patients. Schilling tests were conducted in four patients.

To define the severity of neurological impairment, we used a previously described functional disability scale ${ }^{3}$ : gait disturbance (=unable to maintain the Romberg position, $2=$ impairment, but able to walk unsupported, $3=$ substantial support required for ambulation, $4=$ wheelchair or bed bound); sensory disturbance (hypaesthesia, dysaesthesia, position sense impairment) ( $1=$ impairment only in toes and fingers, $2=$ in ankle and wrists, $3=$ in upper arms and legs), and mental impairment (1=intellectual or behavioural impairment but requires no social support, 2=partially dependent for activities of daily living, $3=$ completely dependent for all activities of daily living). Neuropathy was clinically graded by the loss of reflexes ${ }^{4}$ ( $1=$ loss or reduction of ankle deep tendon reflexes, 2=patellar deep tendon reflexes, $3=$ deep tendon reflexes of the arms) and severity of pyramidal tract damage was graded as $1=$ positive Babinski sign, 2=spastic paraparesis, or $3=$ spastic tetraparesis. The interval between diagnosis and last follow up was 5 to 65 months.

Electroneurographical and EMG studies were performed in all patients, using Amplaid (EMG 15) equipment. Standard techniques 


\begin{tabular}{|c|c|c|c|c|c|c|c|c|c|c|c|c|}
\hline \multirow[b]{2}{*}{ Patient } & \multirow[b]{2}{*}{ Age/sex } & \multirow[b]{2}{*}{ Disease } & \multicolumn{4}{|c|}{ Laboratory findings } & \multicolumn{6}{|c|}{$\begin{array}{l}\text { Clinical findings at admission } \\
\text { (and } 6 \text { months after start of treatment) }\end{array}$} \\
\hline & & & $R B C$ & $M C V$ & Vit $B_{12}$ & $\begin{array}{l}\text { Schilling } \\
(\%)\end{array}$ & Gait & $L t / p p$ & $V i b$ & Pos & Pyrtr & Mental \\
\hline A & $60 / \mathrm{F}$ & Pernicious anaemia & 3.52 & 117.7 & 46 & $\mathrm{ND}$ & $4(2)$ & $3(1)$ & $1(1)$ & $1(1)$ & $2(0)$ & $3(1)$ \\
\hline B & $71 / \mathrm{F}$ & Crohn's disease & 3.76 & 106.9 & $171^{\star}$ & $<9$ & $2(1)$ & $1(1)$ & $2(1)$ & $2(1)$ & $0(0)$ & $0(0)$ \\
\hline $\mathrm{C}$ & $69 / \mathrm{F}$ & Pernicious anaemia & 3.42 & 102.2 & 11 & ND & $3(1)$ & $3(1)$ & $2(1)$ & $2(1)$ & $2(0)$ & $0(0)$ \\
\hline $\mathrm{D}$ & $65 / M$ & Pernicious anaemia & 3.01 & 109 & $202^{\star}$ & 0.40 & $2(1)$ & $2(2)$ & $2(1)$ & $2(0)$ & $0(0)$ & $0(0)$ \\
\hline $\mathrm{E}$ & $67 / M$ & Crohn's disease & 4.41 & 92.6 & 84 & 4.30 & $3(2)$ & $3(3)$ & $2(1)$ & $2(1)$ & $0(0)$ & $0(0)$ \\
\hline $\mathrm{F}$ & $24 / \mathrm{F}$ & Crohn's disease & 3.33 & 130 & 124 & ND & $2(0)$ & $1(1)$ & $1(1)$ & $2(0)$ & $0(0)$ & $0(0)$ \\
\hline G & $79 / \mathrm{F}$ & Gastric resection & 2.97 & 96.2 & 33 & ND & $2(2)$ & $0(0)$ & $2(1)$ & $2(1)$ & $0(0)$ & $0(0)$ \\
\hline $\mathrm{H}$ & $84 / \mathrm{M}$ & Unknown & 3.89 & 89.3 & 128 & ND & $4(-)$ & $2(-)$ & $1(-)$ & $2(-)$ & $3(-)$ & $1(-)$ \\
\hline I & $79 / \mathrm{F}$ & Pernicious anaemia & 3.29 & 130 & 100 & 2.20 & $4(-)$ & $2(-)$ & $2(-)$ & $3(-)$ & $2(-)$ & $2(-)$ \\
\hline
\end{tabular}

^Patients were treated with vitamin $\mathrm{B}_{12}$ before measurement.

Laboratory findings: RBC=erythrocyte counts (normal 4-5.3 million/ul); MCV=mean cell volume (normal 80-100); Vit $\mathrm{B}_{12}=$ serum vitamin $\mathrm{B}_{12}$ concentrations (pg/ $\mathrm{ml}$; normal $>160 \mathrm{pg} / \mathrm{ml}$ ); Schilling test (normal extraction $>9 \%$ ). Clinical findings: disability: no $=0$; mild=1; moderate $=2$; severe $=3$ (only gait: bed/wheelchair bound=4); Lt=light touch; $\mathrm{pp}=$ pin prick; Vib=vibration sense; Pos=position sense; Pyr tr=pyramidal tract damage; Mental = mental impairment.

were applied to measure nerve conduction ${ }^{13}$; EMG was performed with standard concentric needle electrodes. Insertion activity, spontaneous activity, and interference pattern were analysed. A minimum of 10 different needle positions at two different sites in each muscle were studied to detect active denervation.

Somatosensory evoked potentials (SEPs) were elicited by unilateral percutaneous stimulation (0.2 ms square wave pulse) at $3 \mathrm{~Hz}$.
Stimuli were delivered at an intensity just above motor threshold. Medial SEPs (SEP-Med) were recorded from the brachial plexus (Erb potential), cervical spine at C2 (N13), and the contralateral parietal area $2 \mathrm{~cm}$ posterior to $\mathrm{C} 3 / 4$ (N19) with a frontal $(\mathrm{Fz})$ reference. In tibial SEPs (SEP-Tib), active electrodes at the level of the first lumbar vertebra (N22) and at $\mathrm{Cz}$ (P37) were referenced to the iliac crest and $\mathrm{Fz}$ respectively.

A

Before treatment

After treatment

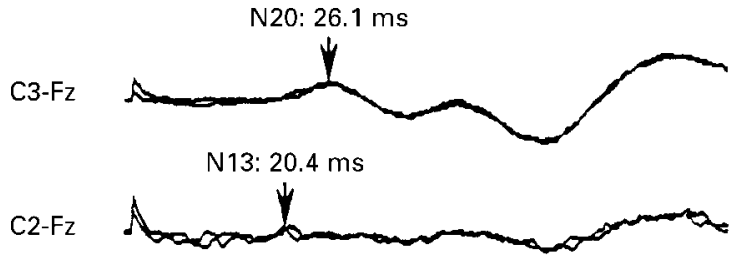

N9: $11.1 \mathrm{~ms}$

EP-Fz

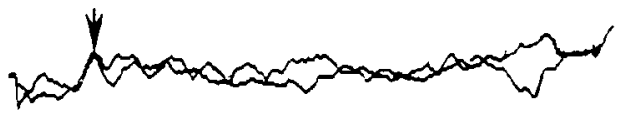

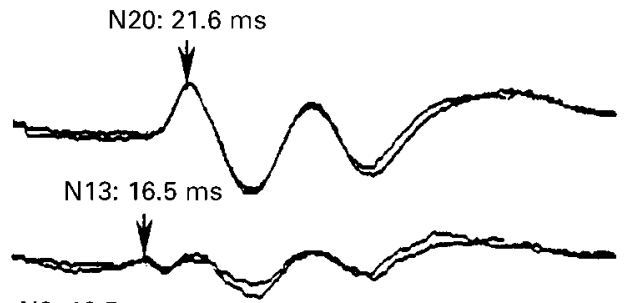

N9: $10.5 \mathrm{~ms}$

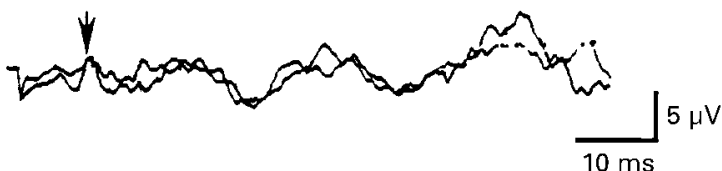

B
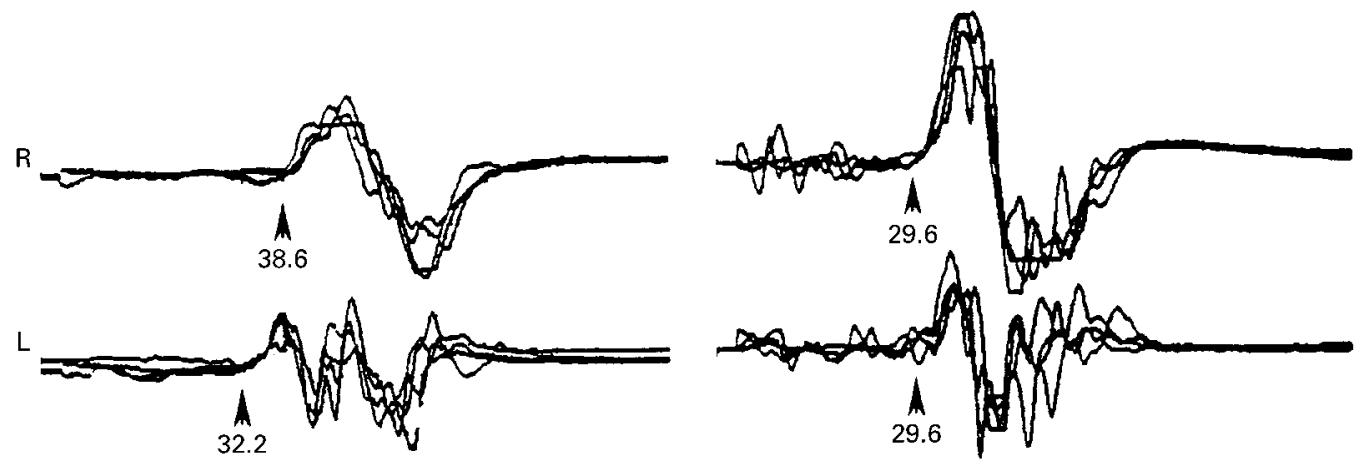

$1 \mathrm{mV}$

$20 \mathrm{~ms}$

Figure 1 (A) Right median nerve somatosensory evoked potentials of patient $A$ before and after treatment with cobalamin. Recordings were from the contralateral scalp $(\mathrm{C} 3-\mathrm{Fz})$ neck $(\mathrm{C2}-\mathrm{Fz})$ ), and Erb's point (EP-Fz). Note normalisation of N13 and N20 after treatment. (B) Motor evoked potentials of patient $C$ recorded from the right $(R)$ and left $(L)$ anterior tibial muscles after transcranial magnetic stimulation of the motor cortex before and after treatment with cobalamin. Lumbar nerve root stimulation was normal at both examinations. Note normalisation of the corticomuscular latencies after treatment. 
Table 2 Somatosensory evoked potentials in SCD

\begin{tabular}{|c|c|c|c|c|c|c|c|}
\hline \multirow[b]{3}{*}{ Patient } & & \multirow[b]{3}{*}{ Neuropathy } & \multicolumn{5}{|c|}{ Before and after substitution with Vitamin $B_{12}$} \\
\hline & & & \multicolumn{3}{|c|}{ Median nerve SEPs } & \multicolumn{2}{|c|}{ Tibial nerve SEPS } \\
\hline & & & N9 (ms) & $\mathrm{N13}$ (ms) & $\mathrm{N} 20$ (ms) & $N 22$ (ms) & P37 (ms) \\
\hline \multirow[t]{2}{*}{ A } & Right & Demyelinating & $11.1(10.5)$ & $20.4(16.5)$ & $26.1(21.6)$ & NR (NR) & $49.2(46.2)$ \\
\hline & Left & Demyelinating & $11.1(10.2)$ & $19.5(16.8)$ & $27.3(22.2)$ & NR (NR) & $53.4(55.2)$ \\
\hline \multirow[t]{2}{*}{ B } & Right & No & 9.6 (ND) & 15.9 (ND) & 21 (ND) & NR (NR) & $46(52.8)$ \\
\hline & Left & No & 10.8 (ND) & 15.9 (ND) & 20.7 (ND) & NR (NR) & $54(53.4)$ \\
\hline \multirow[t]{2}{*}{ C } & Right & No & $11.6(10.5)$ & NR (15.3) & $24.8(21)$ & NR (NR) & $48.8(51)$ \\
\hline & Left & No & $12.8(10.5)$ & NR (NR) & $24(21)$ & NR (25.8) & $65(58.2)$ \\
\hline \multirow[t]{2}{*}{ D } & Right & Mild axonal & 10.8 (ND) & $14.6(\mathrm{ND})$ & 21 (ND) & 22 (NR) & $54(51)$ \\
\hline & Left & Mild axonal & 10.6 (ND) & NR (ND) & $21.4(\mathrm{ND})$ & 22 (NR) & $50(48.6)$ \\
\hline \multirow[t]{2}{*}{$\mathrm{E}$} & Right & Mild axonal & NR (NR) & 15.9 (NR) & $21.6(21)$ & NR (NR) & $55(47.4)$ \\
\hline & Left & Mild axonal & NR (NR) & $15.3(16.5)$ & $21.6(22.2)$ & NR (NR) & $49.8(49.2)$ \\
\hline \multirow[t]{2}{*}{$\mathrm{F}$} & Right & No & $9.7(8.4)$ & NR (12) & $21.6(18.9)$ & NR (18.6) & $61.6(40.8)$ \\
\hline & Left & No & $9.8(8.4)$ & NR (12.3) & $20.8(18.9)$ & NR (18.6) & $58.8(40.2)$ \\
\hline \multirow[t]{2}{*}{ G } & Right & No & NR (ND) & 14.7 (ND) & $22.2(\mathrm{ND})$ & NR (NR) & $48(47.3)$ \\
\hline & Left & No & 10.5 (ND) & 14.7 (ND) & 22.2 (ND) & NR (NR) & $48(49.6)$ \\
\hline \multirow[t]{2}{*}{$\mathrm{H}^{\star}$} & Right & Axonal & 12.3 & 16.2 & 23.4 & 28.2 & 55.2 \\
\hline & Left & Axonal & 11.7 & 17.1 & 23.5 & 28.2 & 56.4 \\
\hline \multirow[t]{2}{*}{$I^{\star}$} & Right & Axonal & ND & 18.4 & 23.6 & NR & 55.2 \\
\hline & Left & Axonal & ND & 17.6 & 23.2 & NR & 49.8 \\
\hline
\end{tabular}

*Patients $\mathrm{H}$ and I died of unrelated diseases before follow up examination could be performed

Numbers in parenthises represent latencies after treatment. $\mathrm{NR}=$ no response; $\mathrm{ND}=$ not done.

Motor evoked potentials (MEPs) were recorded bilaterally with surface electrodes from the hypothenar muscles and the tibialis anterior muscles. During cortical stimulation a slight voluntary contraction of the target muscles was maintained. The shortest latency evoked by at least four stimuli was analysed. Nerve root stimulation was performed at the C7-Th1 or the L4-L5 level, respectively. Latency differences were used to estimate central motor conduction time.

T1 and T2 weighted, sagittal, and axial MRI images of the spine and skull were obtained with a Siemens Magneton impact (1T)) in five patients, a Phillips gyroscan $(1.5 \mathrm{~T})$ in one patient and a Brucker Tomikon $(0.23 \mathrm{~T})$ in one patient. When abnormalities were noted, T1 weighted images were retaken after intravenous administration of contrast medium (gadolinium dimeglumine).

Results

PATIENTS' PROFILE

Six female and three male patients were studied. The underlying diseases causing vitamin $B_{12}$ deficiency are displayed in table 1 . Two patients died of unrelated diseases before the follow up examination. Vitamin $\mathrm{B}_{12}$ deficiency was diagnosed in six patients after admission to our hospital. The remaining patients, diagnosed elsewhere as having haematological disease, developed neurological symptoms as a result of insufficient cobalamin treatment. Laboratory data at the time of clinical presentation are displayed in table 1.

CLINICAL EVALUATIONS

On admission all patients presented gait disturbance, confirmed by the medical examination. Hypaesthesia, dysaesthesia, and impairment of vibration perception or position sense was present in all patients (table 1). Patient $\mathrm{H}$ had hyperactive reflexes of the upper limbs. Patellar tendon reflexes were hyperactive in patient $\mathrm{A}$, reduced in patient $\mathrm{H}$, and absent in patient I. In patients $A, E$, and $G$ ankle reflexes were diminished and in patients $\mathrm{H}$ and
I they were absent. Toes were upgoing in patients $\mathrm{A}, \mathrm{C}, \mathrm{H}$, and I with mild paraparesis (patients A, C, and I) or mild tetraparesis (patient $\mathrm{H}$ ).

Neuropsychological examination disclosed signs of mental impairment with dementia of subcortical type in three patients. Patient $\mathrm{C}$ had depression.

After the diagnosis of vitamin $\mathrm{B}_{12}$ deficiency, intramuscular application of cobalmin was started $(1000 \mu \mathrm{g}$ cobalamin once daily for 4 weeks, then once weekly for 1 year followed by long term monthly treatment). All patients reported clinical improvement after treatment. Patient F had completely recovered. The other patients still had gait disturbance, although all patients were able to walk without help. Patients A, C, D, and E complained of sensory disturbance, especially dysaesthesia of the feet. Physical examinations are summarised in table 1 .

ELECTROPHYSIOLOGICAL DATA

Electroneurography and electromyography

Neurographical and EMG studies disclosed normal results in patients $\mathrm{B}, \mathrm{C}, \mathrm{F}$, and $\mathrm{G}$. Patient A showed markedly slowed sensory nerve conduction velocities and normal motor nerve conduction studies (left $\mathrm{N}$. suralis: 26 $\mathrm{m} / \mathrm{s}, 10 \mu \mathrm{V}$; left $\mathrm{N}$. peroneus: $41 \mathrm{~m} / \mathrm{s}, 4 \mu \mathrm{V}$; left $\mathrm{N}$. ulnaris (sensible Dig V): $39 \mathrm{~m} / \mathrm{s}, 10 \mu \mathrm{V}$; left N. medianus (sensible Dig III): $41 \mathrm{~m} / \mathrm{s}, 20 \mu \mathrm{V}$ ) suggesting a sensory demyelinating neuropathy. Patients D and E had normal motor nerve conduction and only slightly reduced sensory nerve conduction velocities but disproportionally reduced sensory nerve action potentials, suggesting mainly sensory neuropathy of axonal type. Patients $\mathrm{H}$ and $\mathrm{I}$ showed reduced sensory and motor nerve conduction and EMG signs of acute denervation were present in the extensor digitorum brevis and anterior tibial muscles consistent with a sensorimotor neuropathy of mainly axonal type.

Follow up studies were essentially the same except for patient A, whose sensory nerve conduction velocities of the lower limbs improved 

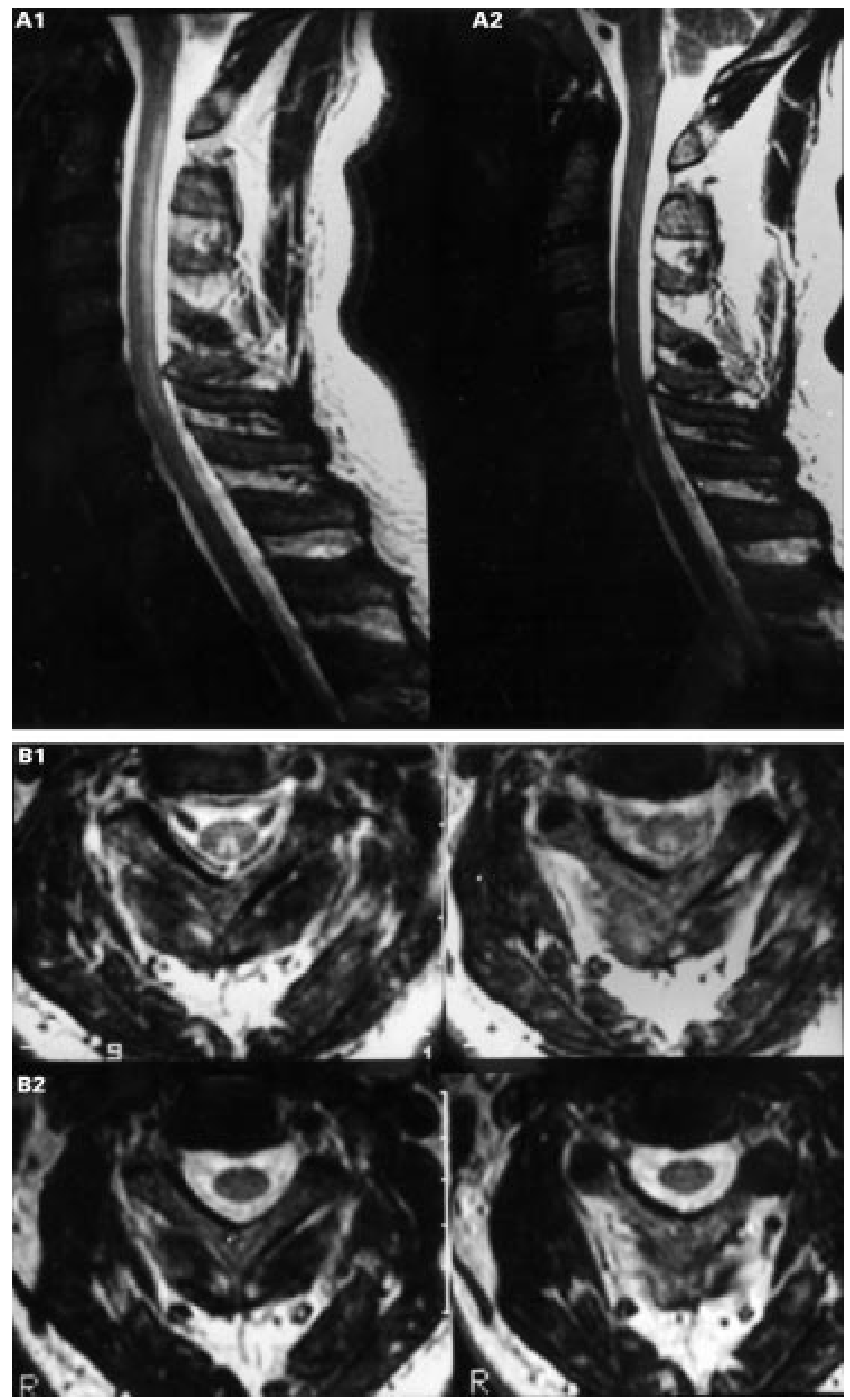

Figure $2 T 2$ weighted sagittal $(A)$ and axial (B) MRI of the cervical and upper thoracic spinal cord of patient D during initial admission with SCD (1) and 12 months after initiation of cobalamin treatment (2). The hyperintense lesions of the posterior column disappeared after treatment. 
from $26 \mathrm{~m} / \mathrm{s}$ to $36 \mathrm{~m} / \mathrm{s}$ and normalised at the upper limbs.

\section{Somatosensory evoked potentials}

Before initiation of treatment, Tib-SEPs were abnormal in all patients with delayed P37 responses and often absence of the lumbar responses. The Med-SEPs were abnormal in all patients except patients D and F. After treatment SEP-Tibs normalised in patient F. In all other patients the P37 response was still delayed. The SEPs-Med normalised in patients $\mathrm{A}$ and $\mathrm{C}$ (fig $1 \mathrm{~A}$, table 2).

Motor evoked potentials

Studies of MEPs were performed in seven untreated patients. Before initiation of treatment, patients A, B, D, E, and G had normal central motor conduction times (CMCTs) to the anterior tibial muscles and to the abductor digiti minimi muscles. Patient $\mathrm{C}$ showed prolonged CMCTs to both anterior tibial muscles and patient $\mathrm{H}$ showed prolongation of the CMCTs to all extremities. After treatment, all patients had normal CMCTs (fig $1 \mathrm{~B}$ ).

MAGNETIC RESONANCE IMAGING

We performed MRI of the spine in patients $A$, $\mathrm{B}, \mathrm{C}$, and $\mathrm{D}$ before and in patients $\mathrm{A}, \mathrm{B}, \mathrm{C}, \mathrm{D}$, $\mathrm{E}$, and $\mathrm{F}$ after treatment. Patients $\mathrm{A}$ and $\mathrm{G}$ underwent MRI of the brain. Before initiation of treatment, patients A and D showed hyperintense lesions within the dorsal columns on $\mathrm{T} 2$ weighted images ranging from $\mathrm{C} 2$ to $\mathrm{C} 7$ (fig 2 1). T1 weighted images were normal and no enhancement was seen after intravenous administration of gadolinium. No abnormalities were found in patient $\mathrm{B}$ and $\mathrm{C}$ using either high field or low field MRI. All MRI examinations of the spinal cord performed after treatment were normal; MRI of patients A and $\mathrm{D}$ (fig 2 2) normalised 8 and 12 months after the initiation of treatment. Patient $G$ had no cerebral abnormalities. Patient A, who was affected by dementia, had diffuse bright lesions around the fourth ventricle on T2 weighted images; no enhancement in $\mathrm{T} 1$ weighted images after gadolinium administration was seen. Although the spinal lesions in this patient resolved, the hyperintense lesions of the brain persisted after 8 months.

\section{Discussion}

Since the beginning of this century, SCD has been a well defined disorder with dysaesthesia, disturbance of position sense, and spastic paraparesis or tetraparesis. The corresponding neuropathological findings are a diffuse, multifocal pattern of axonal loss and demyelination most severe in the cervical and thoracic spinal cord. The disease predominantly affects the posterior columns followed by the anterolateral and anterior tracts. ${ }^{14}$

The pathophysiological mechanism of damage to the nervous system is still not fully understood. Adenosylcobalamin is required as a cofactor for the conversion of methylmalonyl CoA to succinyl CoA. Lack of adenosylcobalamin may lead to accumulation of methylmalonyl CoA, causing a decrease in normal myelin synthesis and leading to incorporation of abnormal fatty acids into neuronal lipids. Alternatively, impaired DNA synthesis could hinder oligodendrocyte growth and thus myelin production. ${ }^{15}$

The clinical profile of our patients was dominated by damage to the posterior column of the spinal cord. The major findings were dysaesthesia and gait disturbance with impairment of position and vibration sense in the lower limbs and often in the upper limbs. The SEP studies disclosed significantly delayed cortical response in most patients accordingly. ${ }^{599}$ Signs of damage to the central motor pathway were present only in four patients, but only two had abnormal MEPs. ${ }^{9}$ This indicates that damage to the pyramidal tract is less frequent and SCD should also be considered in patients with pure afferent fibre damage. Clinical evidence for peripheral neuropathy was found in five patients. Four of them had axonal neuropathy, ${ }^{467}$ but one patient had definite severe sensory demyelinating neuropathy. Neuropsychological manifestations of cobalamin deficiency were seen in three patients.

All patients improved after treatment, but most complained of residual symptoms such as dysaesthesia and gait disturbance. All patients who underwent follow up examination showed residual abnormal findings, mainly impairment of proprioception and mild to moderate dysaesthesia. Two patients still had clinical improvement up to 3 years after onset of cobalamin treatment. Three patients showed little improvement after initial treatment. However, two of them had discontinued the recommended long term treatment schedule. The SEPs-Med normalised after therapy, whereas SEPs-Tib remained abnormal in most patients. Electrophysiological findings in axonal neuropathy were essentially unchanged at follow up examination as reported previously. ${ }^{6}$ By contrast, demyelinating neuropathy resolved in one patient after treatment. This proves that in rare cases vitamin $B_{12}$ deficiency may lead to a primarily demyelinating, reversible neuropathy. ${ }^{16}$

We performed MRI of the spinal cord in four untreated patients and found hyperintense lesions restricted to the posterior column in two of them. Both patients showed clinical and electrophysiological damage to the posterior column and no signs of pyramidal tract involvement. In one patient, MRI of the brain disclosed diffuse hyperintense lesions around the fourth ventricle. After treatment with cobalamin the hyperintense lesions of the spinal cord were resolved after 8 to 12 months. Interestingly no change in the cerebral lesions was found. Corroborating findings by other investigators $^{10-12}$ as well as ours indicate that hyperintense $\mathrm{T} 2$ lesions of the posterior column may be common in SCD, but are not present in every patient. We found no strict correlation between clinical, electrophysiological, and MRI abnormalities in our patients.

In summary, subacute combined degeneration is clinically characterised by predominant affection of the posterior column resulting in 
impairment of position and vibration sense and dysaesthesia. In some patients, MRI shows abnormalities of the spinal cord, indicating demyelination of the posterior column. Involvement of the pyramidal tract and peripheral neuropathy are less pronounced or may be absent. If neuropathy is present, it is mostly axonal and sensory. In rare cases, vitamin $B_{12}$ deficiency can induce demyelinating neuropathy. Normal tibial nerve SEPs strongly argue against the diagnosis of subacute combined degeneration. In general, SCD responds well to adequate treatment. However, full recovery is exceptional and tibial nerve somatosensory evoked potentials usually remain abnormal even after long term treatment.

We thank A Tzou, B Bielekova, and B Gran, Neuroimmunology Branch, NIH for helpful comments on the manuscript.

1 Russel JSR, Batten FE, Collier J. Subacute combined degeneration of the spinal cord. Brain 1900;10:849-50.

2 Lindenbaum J, Healton EB, Savage DG, et al. Neuropsychiatric disorders caused by cobalamin deficiency in the absence of anemia

3 Healton EB, Savage DG, Brust JC, et al. Neurologic aspects of cobalamin deficiency. Medicine (Baltimore) 1991;70: 229-45.
4 Fine EJ, Hallett M. Neurophysiological study of subacute combined degeneration. F Neurol Sci 1980;45:2-3.

5 Krumholz A, Weiss HD, Goldstein PJ, et al. Evoked responses in vitamin B12 deficiency. Ann Neurol 1981;9: 407-9.

6 McCombe PA, McLeod JG. The peripheral neuropathy of vitamin B12 deficiency. $\mathcal{F}$ Neurol Sci 1984;66:117-26.

7 Tomoda H, Shibasaki H, Hirata I, et al. Central versus peripheral nerve conduction. Before and after treatment of subacute combined degeneration. Arch Neurol 1988;45: $526-9$.

8 Soria ED, Fine EJ. Somatosensory evoked potentials in the neurological sequelae of treated vitamin B12 deficiency. Electromyogr Clin Neurophysiol 1992;32:63-71.

9 Di Lazzaro V, Restuccia D, Fogli D, et al. Central sensory and motor conduction in vitamin B12 deficiency. Electroencephalogr Clin Neurophysiol 1992;84:433-9.

10 Berger JR, Quencer R. Reversible myelopathy with pernicious anemia: clinical/MR correlation. Neurology 1991;41: $947-8$.

11 Tracey JP, Schiffman FJ. Magnetic resonance imaging in cobalamin deficiency. Lancet 1992;339:1172-3.

12 Timms SR, Cure JK, Kurent JE. Subacute combined degeneration of the spinal cord: MR findings. A $\mathcal{F}$ Neuroradiol 1993;14:1224-7

13 Kimura J. Electrodiagnosis in diseases of nerve and muscle: principles and practice, Philadelphia: FA Davis, 1989.

14 Pant SS, Asbury AK, Richardson EJ. The myelopathy of pernicious anemia. A neuropathological reappraisal. Acta Neurol Scand 1968;5:1-36.

15 Beck WS. Diagnosis of megaloblastic anemia. Annu Rev Med 1991;42:311-22.

16 Steiner I, Kidron D, Soffer D, et al. Sensory peripheral neuropathy of vitamin B12 deficiency: a primary demyelinating disease? F Neurol 1988;235:163-4. 